Mon. Not. R. Astron. Soc. 000, 000-000 (0000) Printed 24 October $2018 \quad$ (MN LATEX style file v1.3)

\title{
Modelling the Nonlinear Gravitational Clustering in the Expanding Universe
}

\author{
T. Padmanabhan \\ Inter-University Centre for Astronomy and Astrophysics, Post Bag 4, Ganeshkhind, Pune 411007, India \\ email:paddy@iucaa.ernet.in
}

24 October 2018

\begin{abstract}
The gravitational clustering of collisionless particles in an expanding universe is modelled using some simple physical ideas. I show that it is indeed possible to understand the nonlinear clustering in terms of three well defined regimes: (1) linear regime (2) quasilinear regime which is dominated by scale-invariant radial infall and (3) nonlinear regime dominated by nonradial motions and mergers. Modelling each of these regimes separately I show how the nonlinear two point correlation function can be related to the linear correlation function in heirarchical models. This analysis leads to results which are in good agreement with numerical simulations thereby providing an explanation for numerical results. The ideas presented here will also serve as a powerful anlytical tool to investigate nonlinear clustering in different models. Several implications of the result are discussed.
\end{abstract}

Key words: Cosmology : theory - large scale structure of Universe - dark matter structure formation

The driving force behind the formation of large scale structures in the universe is the gravitational field produced by density fluctuations. Overdense regions accrete matter at the expense of underdense regions allowing inhomogeneities in the universe to grow. Observations suggest that the material content of the universe is dominated by dark matter, likely to be made of collisionless elementary particles. In that case, the gravitational force is mainly due to these particles and, to first approximation, we can ignore the complications arising from baryonic physics. The evolution of density perturbations is then governed purely by the gravitational force.

When these density perturbations are small, it is possible to study their evolution using linear theory. But once the density contrast becomes comparable to unity, linear perturbation theory breaks down and one must use N-body simulations to study the growth of perturbations. While these simulations are of some value in making concrete predictions for specific models, they do not provide clear physical insight into the process of non-linear gravitational dynamics. To obtain such an insight into this complex problem, it is necessary to model the gravitational clustering of collisionless particles using simple physical concepts. I shall develop one such model in this paper, which - in spite of extreme simplicity - reproduces the simulation results for hierarchical models fairly accurately. Further, this model also provides insight into the clustering process and can be modified to take into account more complicated situations.

The paradigm for understanding the clustering is based on the well known behaviour of a spherically symmetric overdense region in the universe. In the behaviour of such a region, one can identify three different regimes of interest: (1) In the early stages of the evolution, when the density contrast is small, the evolution is described by linear theory. (2) Each of the spherical shells with an initial radius $x_{i}$ can be parametersed by a mass contained inside the shell, $M\left(x_{i}\right)$, and the energy, $E\left(x_{i}\right)$ for the particular shell. Each shell will expand to a maximum radius $x_{\max } \propto M /|E|$ and then turn around and collapse. Such a spherical collapse and resulting evolution allows a self similar description Filmore and Goldreich 1984 in which each shell acts as though it has an effective radius proportional to $x_{\max }$ Bertschinger 1985. This will be the quasilinear phase. (3) The spherical evolution will break down during the later stages due to several reasons. First of all, non radial motions will arise due to amplification of deviations from spherical symmetry. Secondly, the existence of substructure will influence the evolution in a non-spherically symmetric way. Finally, in the real universe, there will be merging of such clusters [each of which could have been centres of spherical overdense regions in the begining] which will again destroy the spherical symmetry. This will be the nonlinear phase.

The description given above is sufficiently vague and sufficiently well known that one may suspect it can not lead to any insight into the problem. In particular, real universe is hardly spherical. I will show that it is, however, possible 
to model the above process in a manner which allows direct generalisation to the real universe.

To do this we will begin by studying the evolution of system starting from a gaussian initial fluctuations with an initial power spectrum, $P_{i n}(k)$. The fourier transform of the power spectrum defines the correlation function $\xi(a, x)$ where $a \propto t^{2 / 3}$ is the expansion factor in a universe with $\Omega=1$. It is more convenient for our purpose to work with the average correlation function inside a sphere of radius $x$, defined by

$\bar{\xi}(a, x) \equiv \frac{3}{x^{3}} \int_{0}^{x} \xi(a, y) y^{2} d y$

In the linear regime we have $\bar{\xi}_{L}(a, x) \propto a^{2} \bar{\xi}_{i n}\left(a_{i}, x\right)$. In the quasilinear and nonlinear regimes, we would like to have a prescription which relates the exact $\bar{\xi}$ to the mean correlation function calculated from the linear theory. One might have naively imagined that $\bar{\xi}(a, x)$ should be related to $\bar{\xi}_{L}(a, x)$. But one can convince oneself that the relationship is likely to be nonlocal by the following analysis:

Recall that, the conservation of pairs of particles, gives an exact equation satisfied by the correlation function Peebles $19 \$ 0$ :

$\frac{\partial \xi}{\partial t}+\frac{1}{a x^{2}} \frac{\partial}{\partial x}\left[x^{2}(1+\xi) v\right]=0$

where $v(t, x)$ denotes the mean relative velocity of pairs at separation $x$ and epoch $t$. Using the mean correlation function $\bar{\xi}$ and a dimensionless pair velocity $h(a, x) \equiv-(v / \dot{a} x)$, equation (2) can be written as

$\left(\frac{\partial}{\partial \ln a}-h \frac{\partial}{\partial \ln x}\right)(1+\bar{\xi})=3 h(1+\bar{\xi})$

This equation can be simplified by first introducing the variables

$A=\ln a, \quad X=\ln x, \quad D(X, A)=\ln (1+\bar{\xi})$

in terms of which we have Nityananda and Padmanabhan $1 9 9 4 \longdiv { ) }$

$\frac{\partial D}{\partial A}-h(A, X) \frac{\partial D}{\partial X}=3 h(A, X)$

Introducing further a variable $F=D+3 X$, equation (5) can be written in a remarkably simple form as

$\frac{\partial F}{\partial A}-h(A, X) \frac{\partial F}{\partial X}=0$

The characteristic curves to this equation - on which $F$ is a constant - are determined by $(d X / d A)=-h(X, A)$ which can be integrated if $h$ is known. But note that the charecteristics satisfy the condition

$F=3 X+D=\ln \left[x^{3}(1+\bar{\xi})\right]=$ constant

or, equivalently,

$x^{3}(1+\bar{\xi})=l^{3}$

where $l$ is another length scale. When the evolution is linear at all the relevant scales, $\bar{\xi} \ll 1$ and $l \approx x$. As clustering develops, $\bar{\xi}$ increases and $x$ becomes considerable smaller than $l$. It is clear that the behaviour of clustering at some scale $x$ is determined by the original linear power spectrum at the scale $l$ through the "flow of information" along the charesteristics. This suggests that we should actually try to express the true correlation function $\bar{\xi}(a, x)$ in terms of the linear correlation function $\bar{\xi}_{L}(a, l)$ evaluated at a different point.

Let us see how we can do this starting from the quasilinear regime. Consider a region surrounding a density peak in the linear stage, around which we expect the clustering to take place. It is well known that density profile around this peak can be described by

$\rho(x) \approx \rho_{b g}[1+\xi(x)]$

Hence the initial mean density contrast scales with the initial shell radius $l$ as $\bar{\delta}_{i}(l) \propto \bar{\xi}_{L}(l)$ in the initial epoch, when linear theory is valid. This shell will expand to a maximum radius of $x_{\max } \propto l / \bar{\delta}_{i} \propto l / \bar{\xi}_{L}(l)$. In scale-invariant, radial collapse, models each shell may be approximated as contributing with a effective radius which is propotional to $x_{\max }$. Taking the final effective radius $x$ as proportional to $x_{\max }$, the final mean correlation function will be

$\bar{\xi}_{Q L}(x) \propto \rho \propto \frac{M}{x^{3}} \propto \frac{l^{3}}{\left(l^{3} / \bar{\xi}_{L}(l)\right)^{3}} \propto \bar{\xi}_{L}(l)^{3}$

That is, the final correlation function $\bar{\xi}_{Q L}$ at $x$ is the cube of initial correlation function at $l$ where $l^{3} \propto x^{3} \bar{\xi}_{L}^{3} \propto x^{3} \bar{\xi}_{Q L}(x)$. This is in the form demanded by equation (8) if $\bar{\xi} \gg 1$. Note that we did not assume that the initial power spectrum is a power law to get this result.

In case the initial power spectrum is a power law, with $\bar{\xi}_{L} \propto x^{-(n+3)}$, then we find that

$\bar{\xi}_{Q L} \propto x^{-3(n+3) /(n+4)}$

[If the correlation function in linear theory has the powerlaw form $\bar{\xi}_{L} \propto x^{-\alpha}$ then the process desribed above changes the index from $\alpha$ to $3 \alpha /(1+\alpha)$. We shall comment more about this aspect at the end of the paper.]. For the power law case, the same result can be obtained by more explicit means. For example, in power law models the energy of spherical shell will scale with its radius as some power which we write as $E \propto x_{i}^{2-b}$. Since $M \propto x_{i}^{3}$, it follows that the maximum radius reached by the shell scales as $x_{\max } \propto(M / E) \propto x_{i}^{1+b}$. Taking the effective radius as $x=x_{e f f} \propto x_{i}^{1+b}$, the final density scales as

$\rho \propto \frac{M}{x^{3}} \propto \frac{x_{i}^{3}}{x_{i}^{3(1+b)}} \propto x_{i}^{-3 b} \propto x^{-3 b /(1+b)}$

In this quasilinear regime, $\bar{\xi}$ will scale like the density and we get $\bar{\xi}_{Q L} \propto x^{-3 b /(1+b)}$. The index $b$ can be related to $n$ by assuming the the evolution starts at a moment when linear theory is valid. The gravitational potential energy [or the kinetic energy] scales as $E \propto x_{i}^{-(n+1)}$ in the linear theory. This may be seen as follows: The power spectrum for velocity field, $P_{v}(k)$ in the linear regime is related to that of density by $P_{v} \propto P(k) / k^{2} \propto k^{n-2}$. Hence the contribution to $v^{2}$ in each logarithmic scale in $\mathrm{k}$-space is $k^{3} P_{v} / 2 \pi^{2} \propto k^{n+1} \propto$ $x^{-(n+1)}$. Similarly, the gravitational potential energy due to fluctuations is

$\phi \propto \int_{0}^{x} 4 \pi y^{2} d y \frac{\xi(y)}{y} \propto x^{2} \xi(x) \propto x^{-(n+1)}$

So the total energy in the initial configuration scales as $x_{i}^{-(n+1)}$ allowing us to determine $b=n+3$. This shows that the correlation function in the quasilinear regime is the one given by equation (11) . 


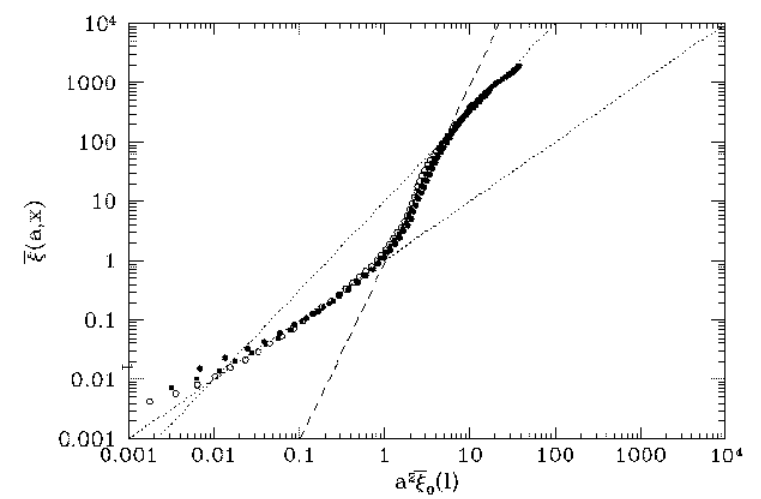

Figure 1. Plot of $\bar{\xi}(a, x)$ against $\bar{\xi}_{L}(a, l)$ for CDM model. The slopes in the three different regimes are indicated. The data points are for three different redshifts [0.1,0.5 and 1.0] and are based on the simulations described in Padmanabhan et al. (1995).

The case with power law initial spectrum has no intrisic scale, if $\Omega=1$. It follows that the evolution has to be self similar and $\bar{\xi}$ can only depend on $q=x a^{-2 /(n+3)}$. This allows us to determine the $a$ dependence of $\bar{\xi}_{Q L}$ by substituting $q$ for $x$ in equation (11). We find

$\bar{\xi}_{Q L}(a, x) \propto a^{6 /(n+4)} x^{-3(n+3) /(n+4)}$

Direct algebra shows that

$\bar{\xi}_{Q L}(a, x) \propto\left[\bar{\xi}_{L}(a, l)\right]^{3}$

reconfirming the local dependence in $a$ and nonlocal dependence in spatial coordinate. This result has no trace of original assumptions [spherical evolution, scale-invariant spectrum ....] left in it and hence once would strongly suspect that it will have far general validity.

Let us now proceed to the third and nonlinear regime. If we ignore the effect of mergers, then it seems reasonable that virialised systems should maintain their densities and sizes in proper coordinates, i.e. the clustering should be "stable". This would require the correlation function to have the form $\bar{\xi}_{N L}(a, x)=a^{3} F(a x)$. [The factor $a^{3}$ arising from the decrease in background density]. From our previous analysis we expect this to be a function of $\bar{\xi}_{L}(a, l)$ where $l^{3} \approx x^{3} \bar{\xi}_{N L}(a, x)$. Let us write this relation as

$\bar{\xi}_{N L}(a, x)=a^{3} F(a x)=U\left[\bar{\xi}_{L}(a, l)\right]$

where $U[z]$ is an unknown function of its argument which needs to be determined. Since linear correlation function evolves as $a^{2}$ we know that we can write $\bar{\xi}_{L}(a, l)=a^{2} Q\left[l^{3}\right]$ where $Q$ is some known function of its argument. [We are using $l^{3}$ rather than $l$ in defining this function just for future convenience of notation]. In our case $l^{3}=x^{3} \bar{\xi}_{N L}(a, x)=$ $(a x)^{3} F(a x)=r^{3} F(r)$ where we have changed variables from $(a, x)$ to $(a, r)$ with $r=a x$. Equation 16 now reads

$a^{3} F(r)=U\left[\bar{\xi}_{L}(a, l)\right]=U\left[a^{2} Q\left[l^{3}\right]\right]=U\left[a^{2} Q\left[r^{3} F(r)\right]\right]$

Consider this relation as a function of $a$ at constant $r$. Clearly we need to satisfy $U\left[c_{1} a^{2}\right]=c_{2} a^{3}$ where $c_{1}$ and $c_{2}$ are constants. Hence we must have

$U[z] \propto z^{3 / 2}$.

Thus in the extreme nonlinear end we should have
$\bar{\xi}_{N L}(a, x) \propto\left[\bar{\xi}_{L}(a, l)\right]^{3 / 2}$

[Another way deriving this result is to note that if $\bar{\xi}=$ $a^{3} F(a x)$, then $h=1$. Integrating equation (f) with appropriate boundary condition leads to equation (23).] Once again we did not need to invoke the assumption that the spectrum is a power law. If it is a power law, then we get,

$\bar{\xi}_{N L}(a, x) \propto a^{(3-\gamma)} x^{-\gamma} ; \quad \gamma=\frac{3(n+3)}{(n+5)}$

This result is based on the assumption of "stable clustering" and was originally derived by Peebles (Peebles 1965). It can be directly verified that the right hand side of this equation can be expressed in terms of $q$ alone, as we would have expected.

Putting all our results together, we find that the nonlinear mean correlation function can be expressed in terms of the linear mean correlation function by the relation:

$$
\bar{\xi}(a, x)=\begin{array}{cl}
\bar{\xi}_{L}(a, l) & \left(\text { for } \bar{\xi}_{L}<1, \bar{\xi}<1\right) \\
\bar{\xi}_{L}(a, l)^{3} & \left(\text { for } 1<\bar{\xi}_{L}<5.85,1<\bar{\xi}<200\right) \\
14.14 \bar{\xi}_{L}(a, l)^{3 / 2} & \left(\text { for } 5.85<\bar{\xi}_{L}, 200<\bar{\xi}\right)
\end{array}
$$

The numerical coefficients have been determined by continuity arguments. We have assumed the linear result to be valid upto $\bar{\xi}=1$ and the virialisation to occur at $\bar{\xi} \approx 200$ which is result arising from the spherical model. The exact values of the numerical coefficients can be obtained only from simulations.

The true test of such a model, of course, is N-body simulations and remarkably enough, simulations are very well represented by relations of the above form. Figure 1 shows the results of a CDM simulation based on the investigations carried out in Padmanabhan et.al (1995). This data can be fitted by the relations Bagla and Padmanabhan 1993) :

$$
\bar{\xi}(a, x)=\begin{array}{cl}
\bar{\xi}_{L}(a, l) & \left(\text { for } \bar{\xi}_{L}<1, \bar{\xi}<1\right) \\
\bar{\xi}_{L}(a, l)^{3} & \left(\text { for } 1<\bar{\xi}_{L}<5,1<\bar{\xi}<125\right) \\
11.2 \bar{\xi}_{L}(a, l)^{3 / 2} & \left(\text { for } 5<\bar{\xi}_{L}, 125<\bar{\xi}\right)
\end{array}
$$

[The fact that numerical simulations show a correlation between $\bar{\xi}(a, x)$ and $\bar{\xi}_{L}(a, l)$ was originally pointed out by Hamilton et al. 1991 who, however, tried to give a multiparameter fit to the data. This fit has somewhat obscured the simple physical interpretation of the result though has the virtue of being very accurate for numerical work.]

A comparison of equations 21) and 22 shows that the physical processes which operate at different scales are well represented by our model. In other words, the processes descibed in the quasilinear and nonlinear regimes for an individual lump still models the average behaviour of the universe in a statistical sense. It must be emphasised that the key point is the "flow of information" from $l$ to $x$ which is an exact result. Only when the results of the specific model are recast in terms of suitably chosen variables, we get a relation which is of general validity. It would have been, for example, incorrect to use spherical model to obtain relation between linear and nonlinear densities at the same location or to model the function $h$. With hindsight, it is clear why such attempts have not succeeded in the past.

It may be noted that to obtain the result in the nonlinear regime, we needed to invoke the assumption of stable clustering which has not been deduced from any fun- 
damental considerations. In case mergers of structures are important, one would consider this assumption to be sus$\operatorname{pect}($ Padmanabhan, Cen, Ostriker and Summers 1995). We can, however, generalise the above argument in the following manner: If the virialised systems have reached stationarity in the statistical sense, the function $h$ - which is the ratio between two velocities - should reach some constant value. In that case, one can integrate equation (6) and obatin the result $\bar{\xi}_{N L}=a^{3 h} F\left(a^{h} x\right)$. A similar argument will now show that

$\bar{\xi}_{N L}(a, x) \propto\left[\bar{\xi}_{L}(a, l)\right]^{3 h / 2}$

in the general case. For the power law spectra, one would get

$\bar{\xi}(a, x) \propto a^{(3-\gamma) h} x^{-\gamma} ; \gamma=\frac{3 h(n+3)}{2+h(n+3)}$

Simulations are not accurate enough to fix the value of $h$; in particular, the asynptotic value of $h$ could depend on $n$ within the accuracy of the simulations. It may be possible to determine this dependence by modelling mergers in some simplified form.

We conclude with two interesting speculations regarding the nonlinear stage. If $h=1$ asymptotically, the correlation function in the extreme nonlinear end depends on the linear index $n$. One may feel that physics at highly nonlinear end should be independent of the linear spectral index $n$. This will be the case if the asymptotic value of $h$ satisfies the scaling

$h=\frac{3 c}{n+3}$

in the nonlinear end with some constant $c$. Only high ressolution numerical simulations can test this conjecture that $h(n+3)=$ constant.

Also note that the radial, scale invariant infall described in the quasilinear regime has the effect of changing the linear correlation function $\bar{\xi}_{L}=x^{-(n+3)}=x^{-b}$ to the quasilinear correlation function $\bar{\xi}_{Q L}=x^{-3 b /(1+b)}$. It is amusing to ask what will be the effect of iterating this process N-times. It is easy to see that the index after $\mathrm{N}$ iterations can be expressed in the form:

$\gamma_{N}=\frac{A_{N} b}{1+B_{N} b} ; \quad A_{N}=3^{N} ; B_{N}=\frac{3^{N}-1}{2}$

The fixed point, of course, is $\gamma_{\infty}=2$ which is the only nontrivial fixed point for such an evolution [with the other, trivial, fixed point being zero]. If one could model the evolution as repeated application of this process, one would expect a continuum of scaling relations with the evolution being driven to a singular isothermal sphere. The quasilinear evolution doesnot change the $x^{-2}$ profile, a result which was noted earlier in Bagla and Padmanabhan (1995). It is not clear whether the clustering can indeed be modelled using equation 26.

The relations obtained in this paper will, of course, have certain limitations on their validity. To begin with, we do expect a weak $n$-dependence in these relations due to averaging over peaks of different heights. This has been discussed using a simple analytic model, as well as numerically, in T. Padmanabhan et.al, (1995) [Also see Mo et.al., 1995) for a similar discussion]. Secondly, the asymptotic behaviour will be sensitive to the value of $\Omega$. When $\Omega<1$, structures "freeze out" during the late stages of evolution and "stable clustering" is likely to be a reasonable assumption. Finally, models like HDM evolve in a manner very different from hierarchical models. In the former, small scale power is generated by the breaking of long wavelength modes and the evolution is best modelled by instability of shell-like regions in the universe. Work is now in progress to generalise the ideas of the present paper for other models.

\section{Acknowledgements}

I thank J.S. Bagla, D. Lynden-Bell, R. Nityananda, J.P. Ostriker and P.J.E. Peebles for several useful discussions. I thank my coauthors R. Cen, J.P. Ostriker and F. Summers for permission to adapt a figure from our joint work. This work was completed when I was visiting Institute of Astronomy, Cambridge in Aug, 95 and I thank Ofer Lahav for hospitality.

\section{REFERENCES}

Bagla J.S., Padmanabhan T., (1993), IUCAA preprint 22/93; Jour. Astrophys. Astronomy (in press)

Bagla, J.S. and Padmanabhan, T. (1995), Evolution of gravitational potential in the quasilinear and nonlinear regimes, IUCAA preprint 8/95; astro-ph 9503077.

Bertshinger, E 1985, Ap. J. Suppl., 58, 1.

Filmore, J.A and Goldreich, P., (1984) Ap. J., 281, 1;

Hamilton A.J.S., Kumar P., Lu E., Matthews A., (1991), Ap. J., 374, L1

Mo, H.J., Jain, B. and White, S.D.M., (1995), preprint.

Nityananda R., Padmanabhan T., (1994), MNRAS., 271, 976

Padmanabhan T., Cen R., Ostriker J.P. and Summers, F.J, (1995), Patterns in gravitational clustering: a numerical investigation; astro-ph 9506051; submitted to $A p . J$.

Peebles, P.J.E, (1965), Ap. J 142, 1317

Peebles, P.J.E. (1980), "The Large-Scale Structure of the Universe" (Princeton: Princeton University Press) 